\title{
High Time-Resolution 640-Gb/s Clock Recovery Using Time-Domain Optical Fourier Transformation and Narrowband Optical Filter
}

\author{
Guan, P.; Mulvad, Hans Christian Hansen; Kasai, K.; Hirooka, T.; Nakazawa, M.
}

Published in:

I E E E Photonics Technology Letters

Link to article, DOI:

10.1109/LPT.2010.2083644

Publication date:

2010

Document Version

Publisher's PDF, also known as Version of record

Link back to DTU Orbit

Citation (APA):

Guan, P., Mulvad, H. C. H., Kasai, K., Hirooka, T., \& Nakazawa, M. (2010). High Time-Resolution 640-Gb/s Clock Recovery Using Time-Domain Optical Fourier Transformation and Narrowband Optical Filter. I E E E Photonics Technology Letters, 22(23), 1735-1737. https://doi.org/10.1109/LPT.2010.2083644

\section{General rights}

Copyright and moral rights for the publications made accessible in the public portal are retained by the authors and/or other copyright owners and it is a condition of accessing publications that users recognise and abide by the legal requirements associated with these rights.

- Users may download and print one copy of any publication from the public portal for the purpose of private study or research.

- You may not further distribute the material or use it for any profit-making activity or commercial gain

- You may freely distribute the URL identifying the publication in the public portal 


\title{
High Time-Resolution 640-Gb/s Clock Recovery Using Time-Domain Optical Fourier Transformation and Narrowband Optical Filter
}

\author{
Pengyu Guan, Student Member, IEEE, Hans Christian Hansen Mulvad, Member, IEEE, \\ Keisuke Kasai, Member, IEEE, Toshihiko Hirooka, Member, IEEE, and Masataka Nakazawa, Fellow, IEEE
}

\begin{abstract}
We present a novel scheme for subharmonic clock recovery from an optical time-division-multiplexing signal using time-domain optical Fourier transformation and a narrowband optical filter. High-resolution 640-Gb/s clock recovery is successfully demonstrated with no pattern dependence. The clock recovery is also applied to a $640-\mathrm{Gb} / \mathrm{s}$ differential phase-shift-keying transmission, and error-free performance is achieved over $300 \mathrm{~km}$.
\end{abstract}

Index Terms-Clock recovery, high-speed optical pulse transmission, optical Fourier transformation, optical time-division multiplexing (OTDM), time-to-frequency conversion.

\section{INTRODUCTION}

C LOCK recovery $(\mathrm{CR})$ is one of the key components in an optical time-division-multiplexing (OTDM) system, where it allows the receiver to synchronize with the data signal at the end of a transmission link. Ultrahigh-speed clock recovery at bit rates of $640 \mathrm{~Gb} / \mathrm{s}$ is very challenging, and it has only been achieved based on some form of nonlinear all-optical processing, such as three-wave mixing in a periodically poled-lithium-niobate device and cross-phase modulation in a semiconductor optical amplifier [1], [2].

Recently, optical signal processing based on time-domain optical Fourier transformation (OFT) has received a lot of attention, because it enables the conversion of an optical waveform between the time and frequency-domains [3]. Several reports have demonstrated the high potential of OFT for ultrahigh-speed OTDM transmission. For example, linear transmission impairments such as jitter and waveform distortions can be eliminated with OFT, by converting the unaltered spectrum profile after transmission into the time-domain [3], [4]. Other applications include pulse-shaping, thus enabling, for example, the timing-jitter tolerant demultiplexing of OTDM data [5].

In this letter, we propose a novel clock recovery scheme using OFT. In the present scheme, a time-domain waveform is transformed into the frequency domain. A similar scheme has already been used for the spectro-temporal imaging of short optical pulses

Manuscript received July 01, 2010; revised September 14, 2010; accepted September 16, 2010. Date of publication October 04, 2010; date of current version November 12, 2010. This work was supported by the JSPS Core-to-Core Program, and the Danish Research Council (FTP Grant 274-08-0055).

P. Guan, K. Kasai, T. Hirooka, and M. Nakazawa are with the Research Institute of Electrical Communication, Tohoku University, Aoba-ku, Sendai 9808577, Japan (e-mail: gpy@ riec.tohoku.ac.jp).

H. C. Hansen Mulvad is with DTU Fotonik, Department of Photonics Engineering, Technical University of Denmark, DK-2800 Kgs. Lyngby, Denmark.

Color versions of one or more of the figures in this letter are available online at http://ieeexplore.ieee.org.

Digital Object Identifier 10.1109/LPT.2010.2083644

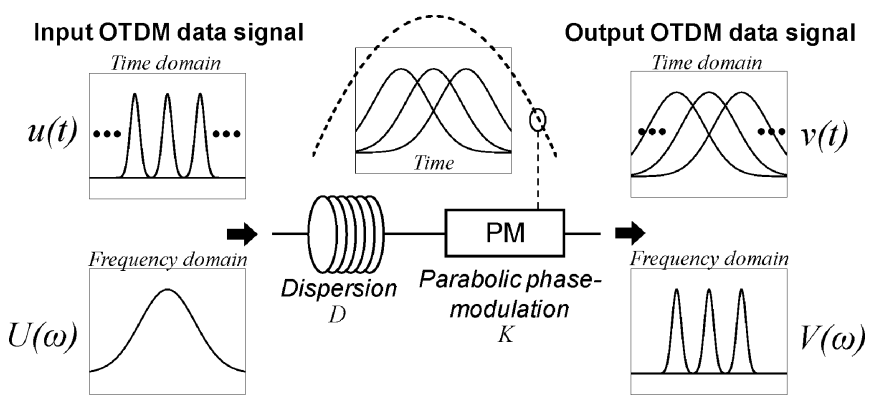

Fig. 1. OFT from time domain to frequency domain.

[6], [7]. Here, the combination of OFT and narrowband optical filtering enables electrooptical phase-comparison with high time resolution between a $40-\mathrm{GHz}$ electrical clock and a $640-\mathrm{Gb} / \mathrm{s}$ OTDM data signal, using lithium-niobate $\left(\mathrm{LiNbO}_{3}\right)$ modulators. As opposed to the earlier 640-Gb/s CR demonstrations, which relied on all-optical phase-comparison in fast-response time devices [1], [2], the present scheme performs a direct electrooptic phase comparison, and hence, does not require an optical pulse source in the CR circuit to achieve the necessary time resolution.

\section{PRINCIPLE}

The principle of OFT for time to frequency domain conversion is shown in Fig. 1. Unlike previous OFT schemes [3]-[5], the waveform is dispersed before the phase-modulation (PM). Consider an input OTDM data signal waveform $u(t)$ and $U(\omega)=$ $\mathcal{F}[u(t)]=\int_{-\infty}^{\infty} u(t) \exp (i \omega t) d t$ is the corresponding spectral profile, consisting of time-interleaved, transform-limited (TL) pulses. First, the pulses are propagated through a group-velocity dispersion (GVD) medium (with $D=k^{\prime \prime} L$, where $k^{\prime \prime}$ is secondorder dispersion and $L$ is length), resulting in broadened waveforms with linear chirp. In the frequency domain, we have

$$
U_{\text {disp }}(\omega)=U(\omega) \exp \left(i \frac{D}{2} \omega^{2}\right) .
$$

The dispersed pulse is then linearly chirped by a $\mathrm{LiNbO}_{3}$ modulator. The output $v(t)$ is expressed as

$$
v(t)=u_{\text {disp }}(t) \exp \left(i \frac{K}{2} t^{2}\right) .
$$

Here, $K$ is the chirp rate. The spectrum of the output pulse is

$$
\begin{aligned}
V(\omega) & =\frac{1}{2 \pi} U_{\operatorname{disp}}(\omega) \otimes \mathcal{F}\left[\exp \left(i \frac{K}{2} t^{2}\right)\right] \\
& =\sqrt{\frac{i}{2 \pi K}} \int_{-\infty}^{+\infty} U_{\operatorname{disp}}\left(\omega^{\prime}\right) \exp \left(-i \frac{1}{2 K}\left(\omega-\omega^{\prime}\right)^{2}\right) d \omega^{\prime}
\end{aligned}
$$




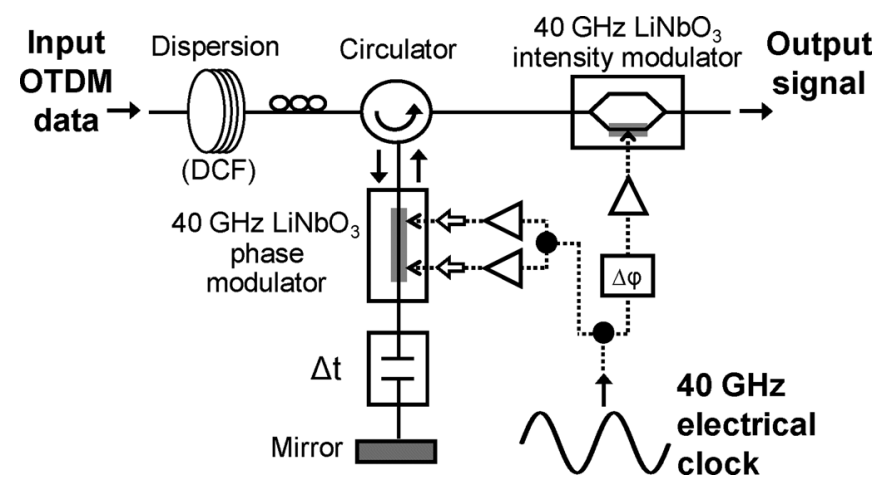

Fig. 2. Setup of OFT used in CR.

where $\otimes$ denotes convolution in the frequency domain to express time-domain multiplication. Equation (3) can be rewritten in the following form when $D=1 / K$ :

$$
\begin{aligned}
V(\omega)= & \sqrt{\frac{i}{2 \pi K}} \\
& \cdot \exp \left(-i \frac{\omega^{2}}{2 K}\right) \int_{-\infty}^{+\infty} U\left(\omega^{\prime}\right) \exp \left[-i\left(-\frac{\omega}{K}\right) \omega^{\prime}\right] d \omega^{\prime} \\
= & \sqrt{2 \pi D i} \exp \left(-i \frac{\omega^{2}}{2 K}\right) u\left(-\frac{\omega}{K}\right)
\end{aligned}
$$

where the integral is equivalent to the inverse Fourier transformation of $U\left(\omega^{\prime}\right)$. Equation (4) indicates that the output spectrum after the OFT is proportional to the waveform $u(t)$. When a pulse with a time offset $\Delta T$ relative to the PM peak, $u_{\Delta T}(t)=u(t-\Delta T)$ (with the corresponding spectrum $\left.U_{\Delta T}(\omega)=U(\omega) \exp (i \omega \Delta T)\right)$, is input to OFT, the output spectrum becomes

$$
V_{\Delta T}(\omega)=\sqrt{2 \pi D i} \exp \left(-i \frac{\omega^{2}}{2 K}\right) u\left(-\frac{1}{K}(\omega-\Delta \omega)\right) .
$$

Here

$$
\Delta \omega=-K \Delta T=-\frac{\Delta T}{D}
$$

is the offset in the frequency domain. Equation (5) indicates that the center frequency of $V_{\Delta T}(\omega)$ depends on $\Delta T$. This means that the different OTDM tributaries are frequency shifted by an amount which depends on the tributary.

\section{Clock Recovery EXPERIMENT}

The OFT setup that we used for the proposed CR scheme is shown in Fig. 2. A short dispersion-compensating fiber (DCF) is used to obtain broadened pulses with linear up-chirp. The PM is achieved by a $40-\mathrm{GHz} \mathrm{LiNbO}_{3}$ phase modulator operated in a round-trip configuration, yielding a chirp rate $K$ up to $0.71 \mathrm{ps}^{-2}$ [4]. The driving signal is a $40-\mathrm{GHz}$ electrical sinusoidal clock. The convex part of the sine is used for PM, which can be approximated by a convex parabola within about $20 \%$ of the repetition period (about 5 ps). Finally, a $40-\mathrm{GHz} \mathrm{LiNbO}_{3}$ intensity modulator is used to suppress the parts of the Fourier-transformed OTDM waveform outside the parabolic PM windows.

First, the OFT is tested using 40-Gb/s pulses (600 fs) as input. The pulses are broadened to $\sim 11$-ps full-width at halfmaximum (FWHM) using a 40-m-long DCF with $D=1.7 \mathrm{ps}^{2}$.
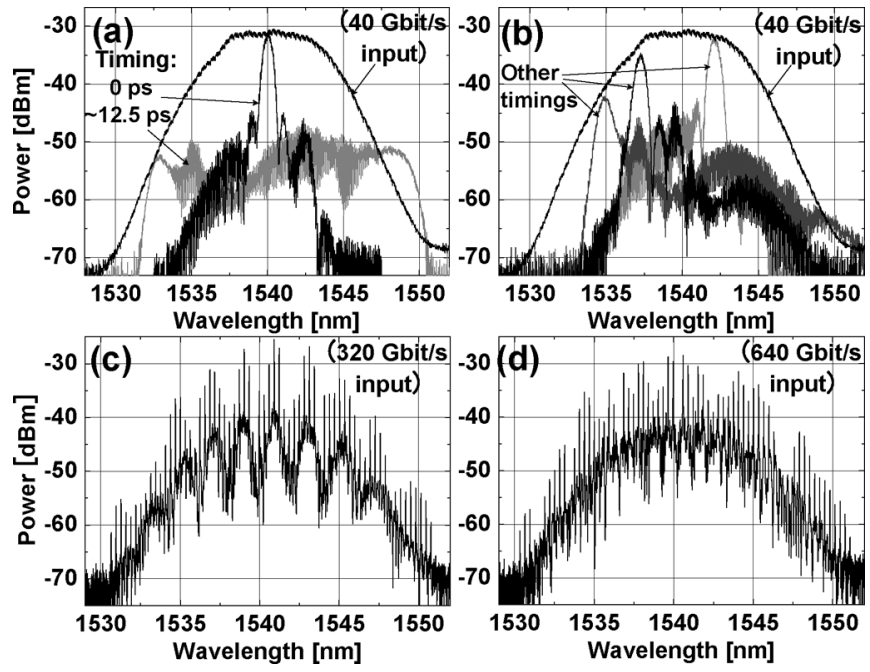

Fig. 3. Output spectra of the OFT (res. $0.01 \mathrm{~nm}$ ). (a) and (b) OFT output spectra for various timing alignments with $40-\mathrm{Gb} / \mathrm{s}$ input. (c) With $320-\mathrm{Gb} / \mathrm{s}$ input. (d) With 640-Gb/s input.

Fig. 3(a) and (b) shows the OFT output spectra for various timing alignments of the input pulse relative to the peak of the phase modulation. The spectral envelope variation in accordance with the time offset is caused by the nonparabolic PM and the adoption of the intensity modulator. The input pulse spectrum with a 6.7-nm FWHM is shown for comparison. These spectra confirm the operation of the OFT, by showing the effect of simultaneous spectral compression and the mapping of tributaries with different timing allocations. Next, 320- and 640-Gb/s OTDM data are used as input to the OFT. The resulting output spectra are shown in Fig. 3(c) and (d), respectively. At $320 \mathrm{~Gb} / \mathrm{s}(\Delta T=3.125 \mathrm{ps})$, the mapping of different tributaries onto wavelengths with $\sim 2.3-\mathrm{nm}$ spacing ( $\Delta \omega=1.8 \mathrm{rad}$. $\mathrm{THz}$ ) is clearly observed. The time-to-frequency conversion ratio is calculated as $\Delta T / \Delta \omega=1.7 \mathrm{ps}^{2}$, which agree with the theoretical value $D=1.7 \mathrm{ps}^{2}$ from (6). At $640 \mathrm{~Gb} / \mathrm{s}$, the spacing is halved, but the tributaries are still discernable.

We characterized the CR operation in a $640-\mathrm{Gb} / \mathrm{s}$ to $300-\mathrm{km}$ transmission. A schematic overview of the experimental setup is shown in Fig. 4. The data transmitter (Tx) is based on a 40-GHz mode-locked fiber laser (MLFL) emitting 2-ps pulses at $1540 \mathrm{~nm}$. The pulses are compressed to a 600-fs FWHM using a 2-km highly nonlinear dispersion-flattened fiber [4]. The pulses are encoded with a 40-Gb/s PRBS pattern using a Mach-Zehnder modulator either by on-off keying (OOK) or differential phase-shift keying (DPSK). The 40-Gb/s data are then multiplexed up to $640 \mathrm{~Gb} / \mathrm{s}$ using an optical delay-line multiplexer, and launched into a $300-\mathrm{km}$ dispersion-managed fiber link. The transmitter setup is described in detail in [4].

On the receiver side, a part of the data signal is input into the clock recovery circuit. The CR circuit is a PLL, as shown in Fig. 4. The OFT is driven by a $40-\mathrm{GHz}$ clock, obtained from a $10-\mathrm{GHz}$ voltage controlled oscillator (VCO) followed by an electrical multiplier $(\times 4)$. The BPF has a 0.2-nm FWHM, which is sufficiently small to resolve the OFT output spectrum at $640 \mathrm{~Gb} / \mathrm{s}$. The locking tributary is selected by changing the time delay between OTDM signal and the 40-GHz RF-signal 


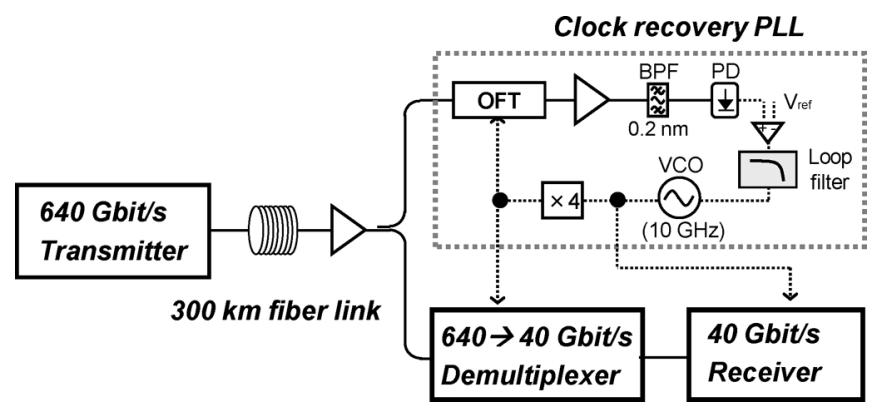

Fig. 4. Experimental setup for $40-\mathrm{GHz} \mathrm{CR}$ in $640-\mathrm{Gb} / \mathrm{s}-300-\mathrm{km}$ transmission.
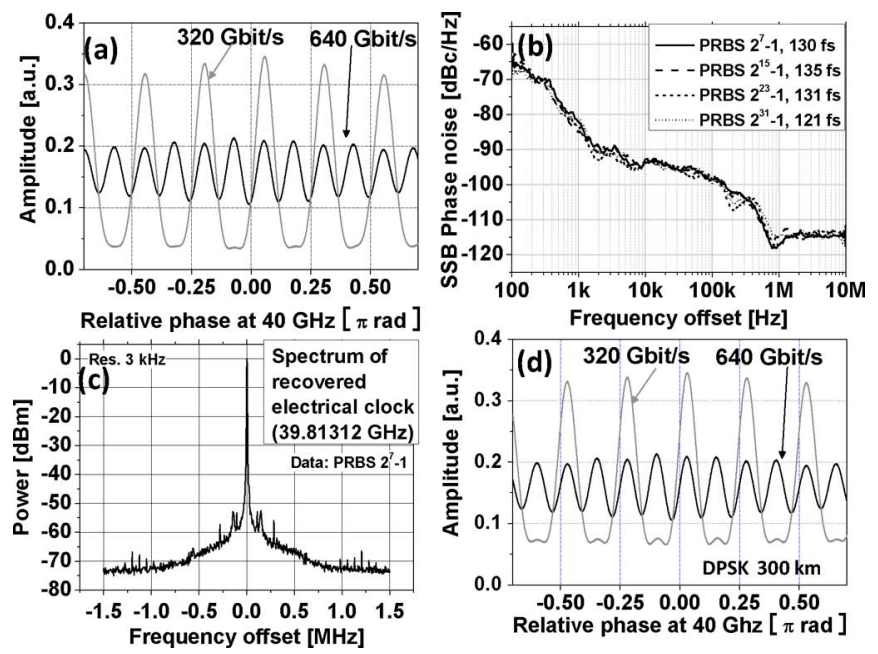

Fig. 5. Clock recovery: (a) Error signals (PD output), (b) 40-GHz clock SSB phase noise for various PRBS (OOK), (c) spectrum of recovered clock. (d) Error signals after $300-\mathrm{km}$ transmission.

driving the OFT. The BPF output is detected by a 1-GHz bandwidth photodiode (PD), followed by a loop filter $(\sim 8-\mathrm{MHz}$ bandwidth) to generate the error signal that controls the VCO phase. The recovered clock triggers a 40-GHz MLFL, which is used as control pulse source for an all-optical semiconductor symmetric Mach-Zehnder (SMZ) switch for demultiplexing. The demultiplexed 40-Gb/s data are injected into a $40-\mathrm{Gb} / \mathrm{s}$ preamplified receiver based on delay-demodulation with a one-symbol delay interferometer (DI), followed by balanced photodetection (BPD). The BPD output is sent to a $40-\mathrm{Gb} / \mathrm{s}$ error-detector, triggered by the recovered clock.

Fig. 5(a) shows the error signal versus the relative phase of the data signal and the 40-GHz RF-signal driving the OFT for 320 and $640 \mathrm{~Gb} / \mathrm{s}$ with an open loop under a back-to-back condition. The traces show a high timing resolution, sufficient to resolve the 640-Gb/s OTDM data. Fig. 5(b) shows single sideband (SSB) phase noise measurements for the $40-\mathrm{GHz}$ clock signal extracted from a $640-\mathrm{Gb} / \mathrm{s}$ OOK signal with various PPG PRBS patterns. By integrating these curves between $100 \mathrm{~Hz}$ and $10 \mathrm{MHz}$, we obtained jitter values in the $\sim 120$ - to 135 -fs range for all PRBSs, thus indicating no pattern dependence. Fig. 5(c) shows the electrical spectra of the recovered clock signal, where the SNR is about $53 \mathrm{~dB}$. Fig. 5(d) shows the error signals at 320 and $640 \mathrm{~Gb} / \mathrm{s}$ after a 300-km DPSK transmission. Compared with Fig. 5(a), almost the same timing resolution is achieved after $300-\mathrm{km}$ transmission.

The BER performance is evaluated by using the $\mathrm{CR}$ in the receiver setup (Rx) shown in Fig. 4. First, the BER performance is

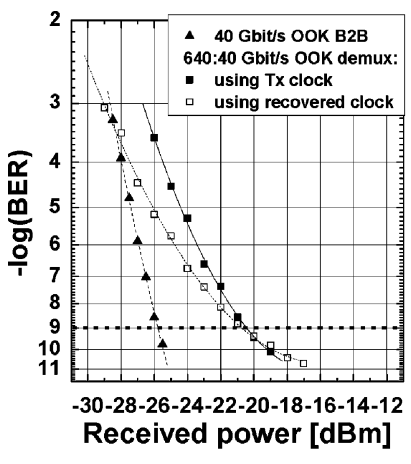

(a)

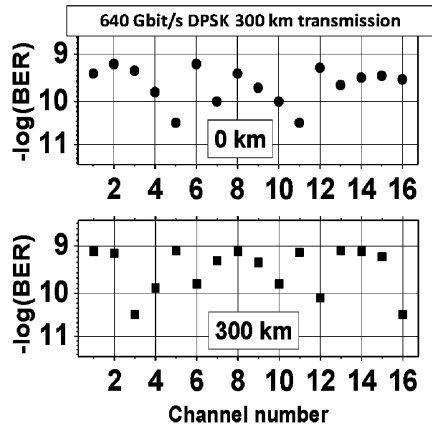

(b)
Fig. 6. BER performance: (a) 640-Gb/s OOK back-to-back; (b) 640-Gb/s DPSK transmission over $300 \mathrm{~km}$ for all OTDM tributaries.

tested under back-to-back conditions with OOK (PRBS $2^{7}-1$ ) data modulation. As shown in Fig. 6(a), error-free performance is achieved when using the CR, and the performance is close to that obtained when using the $40-\mathrm{GHz}$ Tx clock to trigger the Rx. Finally, the CR is tested in a $640-\mathrm{Gb} / \mathrm{s}$ (DPSK) transmission over a 300-km dispersion managed link. Fig. 6(b) shows the BER at a received power of $-23 \mathrm{dBm}$. Error-free performance was achieved for all tributaries, confirming the ability of the CR to successfully retrieve the clock from transmitted data.

\section{CONCLUSION}

We have demonstrated prescaled $40-\mathrm{GHz}$ clock recovery from $640-\mathrm{Gb} / \mathrm{s}$ OTDM data using $\mathrm{LiNbO}_{3}$ modulators, based on time-to-frequency conversion with OFT and optical filtering. The clock recovery was used for error-free demultiplexing and a nearly penalty-free $300-\mathrm{km}$ transmission.

\section{REFERENCES}

[1] E. Tangdiongga, H. C. Hansen Mulvad, H. de Waardt, G. D. Khoe, A. M. J. Koonen, and H. J. S. Dorren, "SOA-based clock recovery and demultiplexing in a lab trial of $640 \mathrm{~Gb} / \mathrm{s}$ OTDM transmission over 50-km fibre link," in Proc. Eur. Conf. Optical Communication (ECOC'07), Berlin, Germany, Sep. 2007, Paper PD1.2.

[2] L. K. Oxenløwe, F. Gomez-Agis, C. Ware, S. Kurimura, H. C. Hansen Mulvad, M. Galili, H. Nakajima, J. Ichikawa, D. Erasme, A. T. Clausen, and P. Jeppesen, "640-Gbit/s data transmission and clock recovery using an ultrafast periodically poled lithium niobate device," J. Lightw. Technol., vol. 27, no. 3, pp. 205-213, Feb. 1, 2009.

[3] M. Nakazawa, T. Hirooka, F. Futami, and S. Watanabe, "Ideal distortion-free transmission using optical Fourier transformation and Fourier transform-limited optical pulses," IEEE Photon. Technol. Lett., vol. 16, no. 4, pp. 1059-1061, Apr. 2004.

[4] T. Hirano, P. Guan, T. Hirooka, and M. Nakazawa, "640 Gbit/s singlepolarization DPSK transmission over $525 \mathrm{~km}$ with time-domain optical Fourier transformation in a round-trip configuration," in Proc. Optical Fiber Communication Conf. (OFC 2010), San Diego, CA, Mar. 2010, Paper OThD7.

[5] L. K. Oxenløwe, M. Galili, A. T. Clausen, and P. Jeppesen, "Generating a square switching window for timing jitter tolerant $160 \mathrm{~Gb} / \mathrm{s}$ demultiplexing by the optical Fourier transform technique," in Proc. Eur. Conf. Optical Communication (ECOC' 06), Cannes, France, Sep. 2006, Paper We2.3.4.

[6] T. Kauffman, W. C. Banyai, A. A. Godil, and D. M. Bloom, "Time-tofrequency converter for measuring picosecond optical pulses," Appl. Phys. Lett., vol. 64, no. 3, pp. 270-272, 1994.

[7] L. K. Mouradian, F. Louradour, V. Messager, A. Barthélémy, and C. Froehly, "Spectro-temporal imaging of femtosecond events," IEEE J. Quantum Electron., vol. 36, no. 7, pp. 795-801, Jul. 2000. 